\title{
SATISFAÇÃO DOS PACIENTES COM A TÉCNICA DE BUTTONHOLE*
}

\author{
Dejanilton Melo da Silva ${ }^{1}$, Jonas Lírio Gurgel ${ }^{2}$, Cristina Lavoyer Escudeiro ${ }^{3}$, Helen Campos Ferreira ${ }^{3}$
}

${ }^{1}$ Enfermeiro. Mestre em Enfermagem. Universidade Federal Fluminense. Niterói, RJ, Brasil.

${ }^{2}$ Profissional de Educação Física. Doutor em Gerontologia Biomédica. Docente do Instituto de Educação Física da Universidade Federal Fluminense. Niterói, RJ, Brasil.

${ }^{3}$ Enfermeira. Doutora em Enfermagem. Docente da Escola de Enfermagem Aurora de Afonso Costa da Universidade Federal Fluminense. Niterói, RJ, Brasil.

RESUMO: Estudo descritivo e exploratório que objetivou investigar os benefícios da técnica de botoeira para canulação de fístula arteriovenosa, durante hemodiálise, na percepção de pacientes submetidos a ela. Foram realizadas entrevistas com quinze pacientes de uma clínica de hemodiálise, localizada no estado do Rio de Janeiro, em maio de 2014, e identificadas quatro temáticas a partir da análise de conteúdo: Dor; Segurança e Qualidade; Estética; e Bem-estar. A técnica de botoeira veio contribuir de forma satisfatória e significativa na qualidade de vida dos pacientes. Eles associaram essa técnica ao baixo nível de dor; à segurança e qualidade, embora não saibam qualificar o tratamento em razão do desconhecimento do funcionamento do processo; à preservação da estética e imagem corporal; e à sensação de bem-estar. Esses resultados apontam para a importância da valorização da opinião e participação do sujeito na escolha de seu tratamento.

DESCRITORES: Insuficiência renal crônica; Diálise renal; Fístula arteriovenosa; Enfermagem.

\section{PATIENT SATISFACTION WITH THE BUTTONHOLE TECHNIQUE}

ABSTRACT: A descriptive and exploratory study was conducted with the aim to investigate the benefits of the buttonhole technique for arteriovenous fistula cannulation, during hemodialysis, in the perception of patients submitted to it. Interviews were conducted with fifteen patients from a hemodialysis clinic in the state of Rio de Janeiro, in May 2014, and four themes were identified from content analysis: Pain; Safety and Quality; Aesthetics; and Well-being. The buttonhole technique has contributed satisfactorily and significantly to the quality of life of patients. They associated this technique with low levels of pain; safety and quality despite not being able to qualify treatment due to the lack of knowledge about the functioning of the process; the preservation of aesthetics and body image; and the sense of well-being. These results point to the importance of valuing the opinion and the subjects' participation in choosing their treatment.

DESCRIPTORS: Chronic renal insufficiency; Renal dialysis; Arteriovenous fistula; Nursing.

\section{SATISFACCIÓN DE LOS PACIENTES CON LA TÉCNICA DE BUTTONHOLE}

RESUMEN: Estudio descriptivo y exploratorio cuya finalidad fue investigar los beneficios de la técnica de Buttonhole para captar fístula arteriovenosa, durante hemodiálisis, en la percepción de pacientes sometidos a ese proceso. Fueron realizadas entrevistas con quince pacientes de una clínica de hemodiálisis, ubicada en el estado de Rio de Janeiro, en mayo de 2014, e identificadas cuatro temáticas con base en el análisis de contenido: Dolor; Seguridad y Cualidad; Estética; y Bienestar. La técnica de Buttonhole contribuyó de forma significativa para la cualidad de vida de los pacientes. Ellos asociaron esa técnica al bajo nivel de dolor; a la seguridad y cualidad, a pesar de que no sabían cualificar el tratamiento en razón del desconocimiento de cómo funciona el proceso; a la preservación de la estética e imagen corporal; y a la sensación de bienestar. Esos resultados apuntan para la importancia de valorar la opinión y participación del sujeto en la elección de su tratamiento.

DESCRIPTORES: Insuficiencia renal crónica; Diálisis renal; Fístula arteriovenosa; Enfermería.

*Artigo extraído da dissertação intitulada: “Estudo retrospectivo da técnica de botoeira em hemodiálise aplicada em usuários do Sistema Único de Saúde (SUS)". Universidade Federal Fluminense, 2014.

Autor Correspondente:

Dejanilton Melo da Silva

Universidade Federal Fluminense

Tv. Alzira Vargas, 48 - 24130210 - Niterói, RJ, Brasil

E-mail: melo_269@hotmail.com
Recebido: 28/05/2015 Finalizado: 07/08/2015 


\section{INTRODUÇÃO}

A técnica ideal para punção da fístula arteriovenosa (FAV) nos pacientes em programa de hemodiálise (HD) crônica ainda não foi estabelecida no Brasil. No entanto, a técnica de canulação mais frequentemente usada é a ropeladder (escada de corda) $)^{(1)}$. Para a sua efetivação, faz-se uma punção venosa em novo sítio, com uso de agulhas cortantes, a cada sessão de diálise ${ }^{(2)}$. Porém, está associada à formação de aneurismas e estenoses por trauma repetitivo no endotélio, cuja progressão compromete a longevidade do acesso vascular ${ }^{(1)}$.

Mais recentemente, uma técnica alternativa vem sendo encorajada por estar associada a menores complicações ao paciente em hemodiálise por fazer uso de agulhas rombas (cegas), introduzidas em um túnel estabelecido previamente a partir da punção em mesmo local, por cerca de dez canulações, fazendo uso de agulha cortante. Trata-se da técnica de buttonhole (botoeira), a qual foi inicialmente utilizada para pacientes com FAV curtas e baixa resistência à dor provocada pela rotatividade das punções ${ }^{(3-4)}$.

A literatura aponta para diversos benefícios da técnica de botoeira em detrimento da tradicional, como: menor sensação de dor durante a canulação; maior facilidade na inserção das agulhas no sítio de canulação; possibilidade de autopunção; e menor probabilidade de desenvolvimento de hematoma $^{(4-5)}$.

Considerando as altas taxas de prevalência e incidência de doença renal crônica (DRC) no Brasil e, portanto, do número cada vez maior de pacientes que dependem de terapias de substituição da função renal para sobreviver, se faz necessário investigar as novas possibilidades terapêuticas considerando a preservação da autonomia e do bem-estar dos pacientes dependentes delas ${ }^{(6-7)}$.

Nesse sentido e levando em conta a escassez de estudos sobre essa temática, especialmente no âmbito nacional, objetiva-se investigar os benefícios da técnica de botoeira para canulação de FAV na percepção de pacientes submetidos a ela.

A relevância desta pesquisa reside em auxiliar profissionais e gestores de saúde na tomada de decisão referente às técnicas associadas ao tratamento da DRC, que atendam às necessidades dos pacientes em todos os seus aspectos.

\section{MÉTODO}

Estudo do tipo descritivo, exploratório de abordagem qualitativa, cujo cenário foi um centro de hemodiálise privado, de atendimento ambulatorial, localizado no município de São Gonçalo, estado do Rio de Janeiro, Brasil.

Os sujeitos da pesquisa foram 15 pacientes, de ambos os sexos, portadores de doença renal crônica, submetidos à técnica de buttonhole para canulação de fístula arteriovenosa para tratamento por hemodiálise, com idade mínima de 18 anos.

Os dados foram coletados por meio de entrevista, a partir de um questionário com questões abertas referentes ao perfil e a opinião dos pacientes quanto à técnica de buttonhole.

As entrevistas ocorreram em maio de 2014, nos períodos da manhã e tarde, na sala de tratamento, durante a hemodiálise para não atrapalhar o andamento da rotina do serviço. Foram gravadas individualmente em equipamento digital, sendo a transcrição realizada no mesmo dia.

Os dados foram tratados por análise de conteúdo temática ${ }^{(8)}$, mediante as seguintes etapas para operacionalização dessa técnica: organização do material; leitura exploratória; categorização por similaridade; e posterior interpretação, inferência e análise crítica do material.

Atendendo às determinações da Resolução 466/2012 do Conselho Nacional de Saúde, a coleta de dados só foi iniciada após parecer favorável do Comitê de Ética em Pesquisa da Universidade Federal Fluminense sob o número 531.731. Antes da coleta de informações, os sujeitos foram convidados e orientados quanto aos objetivos da pesquisa, bem como sobre a necessidade da assinatura do Termo de Consentimento Livre Esclarecido (TCLE), assegurando a eles o direito de acesso aos dados, o anonimato na apresentação dos resultados e a garantia de poderem deixar a pesquisa se assim quisessem.

\section{RESULTADOS}

Dos 15 entrevistados, nove eram homens e seis mulheres, com idade entre 20 e 60 anos e com média de dois anos de terapia hemodialítica. Seis deles mencionaram conhecer, além da técnica de buttonhole, a técnica tradicional de punção.

A partir de cinco questões abertas relativas 
ao uso da técnica de buttonhole, os pacientes se manifestaram livremente e quatro categorias de análise foram estruturadas: Dor; Qualidade e segurança; Estética; e Bem-estar.

\section{Dor}

Falas expressivas mencionadas pelos pacientes em relação ao momento de punção demonstram satisfação com a técnica em questão no que tange à dor:

Doer dói, mas só dói na hora que fura, depois não dói mais. (Paciente 8)

Eu não sinto dor. (Paciente 12)

Sem problemas. Muito boa! Não sinto nem dor. (Paciente 13)

Aqueles que já haviam sido submetidos à técnica tradicional relataram que o uso da punção em locais diferentes provoca mais dor do que quando puncionados em um único local (túnel):

À medida que você vai furando com a cortante no mesmo local, você não vai sentindo mais dores. Seria diferente se você fosse usar uma punção onde cada dia furasse em um local diferente. E isso dói mais! (Paciente 10)

Você quase não sente dor, tanto menos ainda quando você se autopunciona. Mesmo assim, a dor é bem menor do que aquela dor de cada dia puncionar num outro lugar. (Paciente 15)

\section{Segurança e qualidade}

Os entrevistados mostram-se seguros quanto à técnica de buttonhole e a avaliaram como sendo de melhor qualidade quando em comparação com a tradicional:

Eu acho que o button traz mais segurança, mais firmeza. (Paciente 2)

Essa aqui eu acho boa. Acho uma coisa boa. [...] Essa aqui é melhor mil vezes! (Paciente 4)

Eu vim para esta clínica aqui porque soube que tinha a técnica de button. Esta técnica é a melhor que tem. [...] eu não quero fazer a técnica tradicional. Não quero nem saber da tradicional. Não quero! (Paciente 15)

\section{Estética}

A maior parte dos pacientes destacou a importância da estética, e apontou para a importância da preservação da imagem corporal: que ficam com o braço cheio de caroço. (Paciente 3)

Sim, traz de novo a estética, porque eu passo na rua e as pessoas andando do meu lado nem sabem que eu faço hemodiálise. Eu não tenho aqueles hematomas, aquelas coisas grandes que as pessoas ficam olhando. Comigo nunca aconteceu. (Paciente 6)

Pelo menos meu braço não tá encaroçado, né? Aqueles braços grandes, feios. (Paciente 8)

Não cria os caroços que os pacientes antigos têm, reduz os hematomas e você faz uma diálise melhor. (Paciente 9)

Não fica aquela cicatriz, aquele caroço, aqui pelo menos a estética influencia. (Paciente 11)

Não ficam aqueles hematomas. Eu vejo muitas pessoas que têm aquele calombinho no braço, e eu não tenho. Tô fazendo há três anos e, graças a Deus, eu não tenho! (Paciente 14)

\section{Bem-estar}

A sensação de bem-estar de um portador de doença crônica é resultante de um conjunto de fatores que envolvem o tratamento, a aceitação e estabilidade da doença, rede de suporte, autonomia e outros. No que tange ao tratamento da DRC, as técnicas terapêuticas podem ter grande influência, já que elas podem desencadear manifestações estéticas não desejáveis, dor, dificuldade na manutenção das atividades de vida diária, entre outras. Nesta perspectiva, ao serem questionados acerca da sensação global de bem-estar após início do tratamento por meio da técnica de botoeira, os pacientes, em sua maioria, relataram sentirem-se bem, tal como nos discursos a seguir:

Eu tô me sentido muito bem. Me sinto outro, está entendendo? (Paciente 3)

Eu me sinto bem melhor. Acho melhor do que o cateter. (Paciente 5)

Eu me sinto bem. É tranquilo. (Paciente 6)

A princípio eu usava o cateter, então foram cinco meses péssimos. Não tomava banho direito, andava com dificuldade. Enfim, depois que eu passei a usar o botton, eu levo uma vida normal. (Paciente 10)

Eu me sinto bem. Bom, devido ao fato da forma como eu estava e como eu estou hoje, eu me sinto bem. (Paciente 14) 


\section{DISCUSSÃO}

As entrevistas realizadas apontam para a satisfação dos pacientes portadores de DRC submetidos à hemodiálise com a técnica de botoeira no que tange à dor, qualidade e segurança, preservação da estética e bem-estar. Esta percepção foi apreendida tanto por aqueles que conhecem apenas esta técnica quanto por aqueles outrora submetidos à técnica tradicional: escada de corda.

Trata-se de dados importantes, uma vez que a satisfação do paciente com a abordagem terapêutica influencia na adesão ao tratamento e, por conseguinte, nos indicadores de saúde relacionados ao controle da patologia, especialmentenoquetangeàDRCeseu tratamento por meio da hemodiálise, imprescindível para a sobrevivência dos pacientes ${ }^{(9)}$.

Nessa conjuntura, a avaliação da percepção dos pacientes com seu tratamento pode fornecer informações importantes para a reorganização de serviços e para tomada de decisão quanto às terapêuticas e técnicas relacionadas.

A técnica de botoeira, embora descrita pela primeira vez no final da década de $1970^{(4)}$, é ainda pouco utilizada nos serviços de saúde, já que imprime, em curto prazo, maiores custos relacionados aos materiais para efetivação da técnica e treinamento de pessoal, além de ser pouco discutida e estudada e, portanto, menos conhecida.

Estudos internacionais apontam para a supremacia desta técnica quando comparada com a tradicional no que se refere a tempo de hemostasia inferior, menor risco a infiltrações, hematomas, e aneurisma ${ }^{(1,2,10)}$. $\mathrm{E}$, assim como apontado pelos pacientes do presente estudo, a canulação por botoeira imprime uma sensação de dor reduzida ${ }^{(3,11)}$.

Em um estudo com 16 pacientes submetidos à botoeira, foi avaliada a intensidade da dor com uma escala numérica (0 a 10). Durante a fase de tunelização com agulha cortante, a mediana do índice de intensidade da dor foi de 4, e na fase de punção com agulha romba, a mediana foi de $2^{(1)}$. Resultados semelhantes foram apontados por outros estudos ${ }^{(12-13)}$.

Em contrapartida, resultados de um estudo comparativo entre as duas técnicas apontaram para níveis de dor referidos como leve nos dois grupos, no entanto, pacientes submetidos à botoeira experimentaram mais dor, porém menor necessidade de uso de creme anestésico local ${ }^{(14)}$.

Os pacientes entrevistados neste estudo sugerem também quea técnica de botoeira mostrase eficaz e segura, embora não saibam qualificar o tratamento em razão do desconhecimento do funcionamento do processo. Nesta esteira, alguns estudos apontam para menores taxas de complicações relacionadas à botoeira, como hematomas e infiltrações, sugerindo maior eficiência, eficácia e segurança desta técnica quando comparada com o método de escada de $\operatorname{corda}^{(1,4-5)}$.

Algumas complicações relacionadas à FAV podem desencadear alterações estéticas no membro puncionado, acarretando problemas na autoimagem, vergonha, preconceito e depressão, que podem ter efeitos negativos sobre o bem-estar e qualidade de vida do paciente ${ }^{(15)}$. Neste estudo, a punção em casa de botão foi relacionada, pelos entrevistados, à menor probabilidade de alterações na imagem corporal e à manutenção da maioria das atividades de vida diária; percepções que podem contribuir para aderência ao tratamento.

Para um tratamento eficaz, os portadores de DCR precisam seguir recomendações estritas que implicam na mudança de hábitos, especialmente alimentares e aos relacionamentos à ingestão de medicamentos e comparecimento às sessões de hemodiálise, que geralmente precisam ser realizadas três vezes por semana. Esses pacientes estão propensos a eventos adversos relacionados à terapia, além de problemas psicossociais inerentes às modificações de vida, que podem afetar as dimensões familiar, laboral, amorosa, e à autopercepção dada às possibilidades de alteração de imagem, menor autonomia e independência para desenvolvimento de atividades de vida diária ${ }^{(3,9-16)}$.

Nesse sentido, é preciso valorizar a opinião do sujeito; avaliar ações terapêuticas a partir da escuta sensível que contribua para compreensão das necessidades cotidianas da pessoa com $D C^{(16)}$, entendendo que o cuidado aos usuários de unidades de hemodiálise deve seguir uma perspectiva de atenção ampliada que contemple suas demandas de saúde, uma vez que é cenário de potencialidades para efetivação da prática de integralidade em saúde ${ }^{(17)}$.

\section{CONSIDERAÇÕES FINAIS}

A intenção deste trabalho foi apreender a percepção do portador de insuficiência renal 
atribuída à técnica de botoeira para canulação de fístula durante a hemodiálise. Os depoimentos revelam que a técnica de botoeira veio contribuir de forma satisfatória e significativa na qualidade de vida dos pacientes. Eles associaram essa técnica ao baixo nível de dor, à segurança e qualidade, à preservação da estética e imagem corporal e à sensação de bem-estar. Esses resultados apontam para importância da valorização da opinião e participação do sujeito na escolha de seu tratamento.

Este estudo encontra limitação na profundidade da discussão dada a escassez de literatura que considere a percepção de pacientes quanto ao tratamento por hemodiálise. Não obstante, poderá subsidiar novos estudos que avaliem a técnica de canulação em destaque a fim de contribuir para a prática de enfermagem e para o sucesso da terapêutica.

\section{REFERÊNCIAS}

1. Castro MCM, Silva CF, Souza JMR, Assis MCSB, Aoki MVS, Xagoraris $M$, et al. Punção da fístula arteriovenosa com a técnica em casa de botão com agulha romba. J. Bras. Nefrol. [Internet] 2010;32(3) [acesso em 10 nov 2014]. Disponível: http://dx.doi.org/10.1590/S010128002010000300010

2. Muir CA, Kotwal SS, Hawley CM, Polkinghorne K, Gallagher MP, Snelling P, et al. Buttonhole cannulation and clinical outcomes in a home hemodialysis cohort and systematic review. Clin. J. Am. Soc. Nephrol. [Internet] 2014;9(1) [acesso em 16 mai 2015]. Disponível: https://dx.doi.org/10.2215/CJN.03930413

3. Silva GST, Silva RA, Nicolino AM, Pavanetti LC, Alasmar VL, Guzzardi R, et al. Experiência inicial com a Técnica de buttonhole em um centro de hemodiálise Brasileiro. J. Bras. Nefrol. [Internet] 2010;32(3) [acesso em 03 mar 2011]. Disponível: http://dx.doi.org/10.1590/ S0101-28002010000300006

4. Twardowski Z, Kubara H. Different sites versus constant sites of needle insertion into arteriovenous fistulas for treatment by repeated dialysis. Dial Transplant 1979;(8):978-80.

5. Ball LK. A Técnica de buttonhole para canulação de fístula arteriovenosa. Nephrol. Nurs. J. [Internet] 2006;33(3) [acesso em 03 mar 2011]. Disponível: http://www.nipro.com.br/wp-content/themes/nipro/ catalogos/artigo_Dull.pdf

6. Sesso RC, Lopes AA, Thomé FS, Lugon JR, Watanabe Y, Santos DR. Relatorio do Censo Brasileiro de Dialise Cronica 2012. J. Bras. Nefrol. [Internet] 2014;36(1) [acesso em 18 jun 2015]. Disponível: http://dx.doi. org/10.5935/0101-2800.20140009
7. Mendonça AEO, Dantas JG, Andrade DA, Segato CT, Torres GV. Perfil sociodemográfico e clínico de idosos submetidos à Hemodiálise. Cogitare Enferm. [Internet] 2015;20(1) [acesso em 16 mai 2015]. Disponível: http:// dx.doi.org/10.5380/ce.v20i1.37080

8. Bardin L. Análise de conteúdo. São Paulo: Edições 70; 2011.

9. Silva GM, Gomes IC, Machado EL, Rocha FH, Andrade EIG, Acurcio FA, et al. Uma avaliação da satisfação de pacientes em hemodiálise crônica com o tratamento em serviços de diálise no Brasil. Physis. [Internet] 2011;21(2) [acesso em 16 mai 2015]. Disponível: http:// www.scielo.br/scielo.php?script=sci_arttext\&pid =S0103-73312011000200013

10. Pueyo CG, Navarrete IG, Mejía CM, Blanco MG, García-Ciaño XV, Vaca JR, et al. La punción del acceso vascular en hemodiálisis es una necesidad, el método Buttonhole una opción. Rev. Soc. Esp. Enferm. Nefrol. [Internet] 2011;14(1) [acesso em 10 nov 2014]. Disponível: http://dx.doi.org/10.4321/S113913752011000100005

11. Atkar RK; MacRae JM. The buttonhole technique for fistula cannulation: pros and cons. Curr. Opin. Nephrol. Hypertens. [Internet] 2013;22(6) [acesso em 10 nov 2014]. Disponível: http://dx.doi.org/10.1097/ MNH.0b013e328365ae9e

12. Ludlow V. Buttonhole cannulation in hemodialysis: improved outcomes and increased expense--is it worth it?. CANNT J. [Internet] 2010;20(1) [acesso em 16 mai 2015]. Disponível: http://www.ncbi.nlm.nih.gov/ pubmed/20426358

13. Verhallen AM, Kooistra MP, van Jaarsveld BC. Cannulating in haemodialysis: Rope-ladder or buttonhole technique?. Nephrol. Dial. Transplant. [Internet]2007;22(9) [acessoem16mai2015].Disponível: http://www.ncbi.nlm.nih.gov/pubmed/17557776

14. van Loon MM, Goovaerts T, Kessels AGH, van der Sande FM, Tordoir JHM. Buttonhole needling of haemodialysis arteriovenous fistulae results in less complications and interventions compared to the ropeladder technique. Nephrol. Dial. Transplant. [Internet] 2010;25(1) [acesso em 16 mai 2015]. Disponível: http:// www.ncbi.nlm.nih.gov/pubmed/19717827

15. Almeida AM, Meleiro AMAS. Depressão e insuficiência renal crônica: uma revisão. J. Bras. Nefrol. 2000;22(1):192-200.

16. Pereira LP, Guedes MVC. Hemodiálise: a percepção do portador renal crônico. Cogitare Enferm. [Internet] 2009;14(4) [acesso em 16 mai 2015]. Disponível: http:// dx.doi.org/10.5380/ce.v14i4.16384

17. Fujii CDC, Oliveira DLLC. Factors that hinder of integrality in dialysis care. Rev. Latino-Am. Enfermagem. 
[Internet] 2011;19(4) [acesso em 18 jun 2015] Disponível:

http://dx.doi.org/10.1590/S0104-11692011000400014 